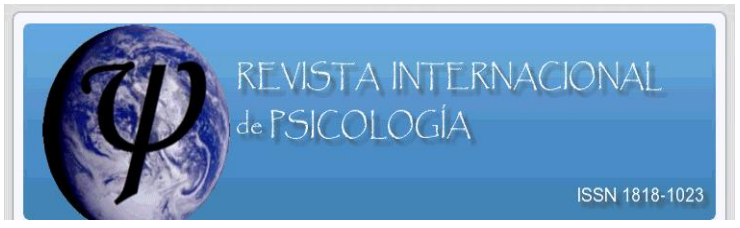

\title{
ESTRÉS LABORAL, CONSIDERACIONES SOBRE SUS CARACTERÍSTICAS Y FORMAS DE AFRONTAMIENTO
}

\author{
Roberto Rodríguez González, Yamila Roque Doval, Osana Molerio Pérez ${ }^{1}$
}

\begin{abstract}
Resumen
El presente trabajo al abordar el estrés y en particular su vinculación con la problemática laboral, intenta además de presentar las experiencias y recopilaciones de información de los autores, brindar enfoque novedoso e integral de 1 mismo atendiendo a la presencia e importancia que gana en la modernidad. Se ofrecen además recomendaciones acerca de su enfrentamiento desde los planos personal y organizacional.
\end{abstract}

\section{Palabra claves}

estrés, estrés laboral, organización laboral

\section{Introducción}

El trabajo fue y es utilizado por el hombre para satisfacer sus necesidades a partir de las acciones sobre el ambiente, y como no siempre puede hacerlo solo, se reúne con otros de sus semejantes para de forma coordinada, lograr los objetivos. Se crean de esta manera las organizaciones, estas son infinitamente variables. Si se desea trabajar con ellas o dirigirlas, es necesario comprender su funcionamiento y estructura, por lo que resulta importante definir teóricamente los elementos que las caracterizan, permiten estudiarlas e intervenir en ellas.

Las organizaciones son algo más que la simple suma de sus partes. Exige considerarlas como sistemas complejos debido al alto nivel de integración y complejidad entre componentes para lograr los objetivos propuestos y su permanencia mediante el mantenimiento de su estabilidad interna. En la actualidad las organizaciones están inmersas en un medio muy inestable debido entre otros factores al rápido desarrollo de la tecnología, la expansión, que implica auge de la mercadotecnia, y a los rápidos cambios sociales, económicos y políticos.

El enfoque de una organización desde el punto de vista sistémico hace necesario identificar sus recursos fundamentales. De los que Chiavenatto (1992) señala cinco:

Recursos materiales de producción: conformado por los edificios, fábricas, maquinaria, equipos, materiales, instalaciones, materias primas, así como por el proceso productivo y la tecnología.

Recursos financieros: constituidos por el capital, la facturación, las inversiones, préstamos, financiación y crédito. Así como también los aspectos contables y presupuéstales, tesorería y valoraciones bancarias.

Recursos de mercadotecnia: nombrados, en ocasiones recursos comerciales, incluyen en su consideración más amplia las ventas, la promoción, propaganda, distribución, la asistencia técnica, las relaciones con el mercado y la investigación de mercadeo.

\footnotetext{
${ }^{1}$ Universidad Central "Marta Abreu " de Las Villas. Correo electrónico: robertor@uclv.edu.cu
} 
Recursos administrativos: que integran las distintas partes y elementos de la organización en sí, así como la de integración ésta a su entorno, incluye todos los subsistemas de toma de decisiones y de comando de la organización.

Recursos humanos (RH): que incluyen a todos los miembros de la organización, en todos sus niveles y subsistemas.

El destino de la organización depende en gran parte de sus recursos humanos, si estos son capaces, pueden enfrentar con éxito la competencia y aprovechar al máximo los recursos técnicos y materiales de que se dispone, entonces la organización podrá lograr sus objetivos, por tanto los recursos humanos constituyen el recurso estratégico.

Presentes en todos los subsistemas de la organización, los recursos humanos, constituyen el elemento esencial dentro de ésta.

El factor humano no ha sido considerado de la misma forma consecuentemente los teóricos y prácticos que de él se han ocupado no lo han valorado de igual modo.

Actualmente, cuando parece estar lejos la concepción del factor humano como factor de producción minimizando su costo, se intenta considerarlo como el recurso "motor" que permite incrementar la competitividad de la organización, logrando sinergia entre lo económico y lo social. En fin se consideran como "capital humano" en el cual se invierte cada día más.

Aceptada su trascendencia, resulta de vital importancia la gestión de los recursos humanos, entendiendo por ella el sistema que instrumenta el procedimiento para el adecuado tratamiento de los mismos, que resulta clave para la organización.

La gestión de recursos humanos es el conjunto de acciones encargada de lograr la visión y atención integral de los mismos considerándolos como estratégicos en y para cada área. Significa conquistar y mantener a las personas en la organización, trabajando y dando el máximo de sí, con actitud positiva y favorable, además, debe constituir un punto clave para gestionar la interacción entre las aptitudes, intereses, necesidades, motivación, talento, expectativas, hábitos y valores del ser humano con la cantidad y calidad de resultados y la satisfacción que la persona pueda conseguir de su trabajo, por ello debe crear un medio que aproveche esas cualidades logrando una comunicación y acción tal que permita alcanzar los objetivos y fines tanto individuales como organizacionales, entre ellos se debe enmarcar el propio hombre como ser en sus aspectos sociales, psicológicos y biológicos. Por tanto la gestión de recursos humanos debe contribuir a la satisfacción laboral y calidad de vida de los recursos humanos.

La gestión de los recursos humanos conforma un sistema de distintas actividades en forma de subsistemas interdependientes estrechamente relacionados, aspecto que hace que cualquier alteración que ocurra en uno, provoque influencia sobre los demás, con las consecuentes alteraciones en los mismos. Es también un sistema abierto, ya que es influido y depende en gran medida del entorno, por ello su carácter contingencial o situacional, o sea, dependen de la situación organizacional; del ambiente, de la tecnología empleada por la organización, de las políticas y directrices vigentes, de la filosofía administrativa preponderante, de la concepción que existe en la organización sobre el hombre y su naturaleza y sobre todo de la cantidad y calidad de los recursos humanos disponibles. 
$\mathrm{Al}$ gestar los recursos humanos es necesario tener en cuenta diversos factores dinámicos entre los que se destacan las edades de las personas que ocupan los diferentes puestos, el aumento o reducción de las cantidades de puestos y la velocidad con que el personal abandona la empresa cualquiera que sea la razón para ello, alteraciones en los estados de salud tanto en aspectos biológicos-fisiológicos como psicológicos y cualquier otra incidencia en la calidad de vida, así como los factores externos que influyen en ésta, tales como el nivel de educación, las actividades prevalecientes en la sociedad (como la actitud ante el trabajo), las numerosas leyes y reglamentos que afectan de forma directa a la organización, las condiciones económicas y la disponibilidad y demanda del personal.

Las alteraciones que se produzcan en cualquiera de los componentes de la organización pueden generar situaciones susceptibles de actuar como agentes estresante, es por ello que el estrés resulta (además de una afectación a los recursos humanos que repercute en los resultados de su actividad), una alerta para la organización en el sentido de que "algo" no está funcionando de manera adecuada en la misma y por tanto es necesario solucionarlo para evitar afectaciones en su recurso más preciado, el humano. Es por ello que en la gestión de recursos humanos se enmarca la atención a la seguridad y salud ocupacional.

\section{La problemática mundial de la salud ocupacional.}

En la actualidad cerca del $45 \%$ de la población mundial y el 58\% de la población mayor de 10 años constituye la denominada "fuerza laboral"; su trabajo sustenta la economía y crea la base material de la sociedad. La salud ocupacional y el bienestar es prerrequisito para la productividad, constituyendo el factor más importante, inclusive, en el aspecto socioeconómico y el desarrollo sostenible.

El lugar de trabajo puede constituir un ambiente peligroso para la seguridad y la salud ocupacional para gran número de trabajadores en los diferentes sectores de la economía. Aproximadamente entre el $30 \%$ y 50\% de los trabajadores reportan estar expuestos a altos riesgos físicos, químicos, biológicos, inexplicables sobrecargas de trabajo pesado además de factores ergonométricos que son una amenaza para su salud y su capacidad de trabajo.

Se estima que anualmente cerca de 120 millones de accidentes de trabajo se traducen en cerca de 200,000 fatalidades y entre 68 y 157 millones de nuevos casos de enfermedades ocupacionales son causados por explosión directa en algunos lugares de trabajo. En adición a todo este panorama, se suma un costo extremadamente alto equivalente, en algunos países a varios puntos en el porcentaje del producto interno bruto (PIB). 
Tendencias de las economías mundiales

Los avances de la ciencia conducen a nuevos desarrollos en los sistemas de producción. Igualmente, los adelantos de la tecnología, en información, automatización, mayor mecanización, nuevos materiales, producción creciente, uso de productos químicos, implementación de biotecnología a escala industrial, y las estrategias industriales de reciclamiento significan gran impacto en el ambiente de trabajo.

En resumen la segunda revolución industrial, provoca notable impacto sobre las condiciones de trabajo y la salud ocupacional.

Asegurar la salud y seguridad de los trabajadores como también la salud ambiental debe ser considerado clave para el desarrollo continuado de las nuevas tecnologías.

Su repercusión sobre la salud ocupacional será probablemente positivo para controlar y prevenir exposiciones de orden físico, químico, biológico y mecánico. Al mismo tiempo las exigencias de los nuevos empleos, la necesidad de analizar la información y varias actividades anexas pueden incrementar problemas psicológicos del trabajo tal como el estrés mental.

\section{Situación y amenazas en la salud ocupacional y seguridad industrial}

La salud ocupacional de los trabajadores y los lugares de trabajo varían sustancialmente de acuerdo con las estructuras económicas, los niveles de industrialización, estado de desarrollo, condiciones climáticas y tradición de salud y seguridad ocupacional. Del 20 al 50\% de los trabajadores pueden estar expuestos a peligros de trabajo en los países industrializados. Este porcentaje puede ser mucho más alto en los países en desarrollo y los recién industrializados, riesgos mecánicos y físicos como también agentes químicos son los principales problemas en la manufactura industrial.

Por ejemplo, los plaguicidas, el trabajo físico pesado, tierras orgánicas, factores biológicos y los accidentes son la preocupación principal de los trabajadores de la agricultura.

Atendiendo a los numerosos problemas de la salud en el trabajo y la cantidad de trabajadores, la necesidad de implementar programas de salud ocupacional es evidente. El tipo de problema puede variar sustancialmente de acuerdo con las necesidades nacionales y locales, influencias culturales y otros factores.

Particular manifestación tiene la problemática de la mujer incrementado por la denominada doble jornada (atención del hogar además de la responsabilidad laboral), la maternidad y manifestaciones de discriminación que implican incluso menor retribución por igual trabajo. En algunos casos resultan expuestas a violencia o acoso sexual. 


\section{Objeto de la salud ocupacional.}

Tiene por objeto el mejoramiento y mantenimiento de las condiciones de vida y salud de la población trabajadora.

Prevenir todo daño para la salud de las personas, derivada de las condiciones de trabajo.

Proteger a las personas contra los riesgos relacionados con agentes físicos, biológicos, químicos, psicosociales, mecánicos, eléctricos y otros derivados de la organización laboral que puedan afectar la salud individual o colectiva en los lugares de trabajo.

Eliminar o controlar los agentes nocivos para la salud integral del trabajador en los lugares de trabajo.

Proteger la salud de los trabajadores y de la población contra los riesgos causados por las radiaciones.

Proteger a los trabajadores y a la población contra los riesgos para la salud provenientes de la producción, almacenamiento y transporte, expendio, uso de sustancias peligrosas para la salud pública.

\section{Campo de aplicación:}

Se aplicará como componente de la gestión de los recursos humanos de la organización, cualquiera que sea la forma jurídica y el objetivo de esta, regula las acciones destinadas a promover y proteger la salud de las personas.

Programa de salud ocupacional en las empresas

Requisitos:

El programa será de carácter permanente.

El programa estará constituido por 4 elementos básicos: Actividades de medicina preventiva. Actividades de medicina de trabajo. Actividades de higiene y seguridad industrial. Funcionamiento del comité de medicina, higiene y seguridad industrial en la empresa.

Las actividades de medicina preventiva, medicina de trabajo e higiene y seguridad industrial serán programada y desarrolladas en forma integrada. Su contenido y recursos deberán estar en directa relación con el riesgo potencial y con el número de trabajadores en el lugar de trabajo.

La organización y el funcionamiento se harán conforme a las reglamentaciones que expidan los ministerios de salud, trabajo y seguridad social.

Formas de los programas de salud ocupacional

Los programas dentro de las empresas podrán ser realizados de acuerdo a las siguientes alternativas.

Exclusivos y propios para la empresa: En conjunto con otras empresas. Contratos con una entidad que preste tales servicios, reconocida por el ministerio de Salud para tales fines. 
Contenido de los programas de salud ocupacional

Los programas de salud ocupacional de las empresas deberán contener:

El subprograma de medicina preventiva comprenderá las actividades que se derivan de los artículos 125, 126 y 127 de la ley $9^{a}$ de 1979, y todas las que estén bajo la asesoría del instituto colombiano de la juventud y el deporte.

- El subprograma de Medicina de Trabajo de la empresa deberá:

Realizar exámenes médicos, clínicos y para clínicos para la admisión, selección de personal ubicación según aptitudes, cambios de ocupación, reingreso al trabajo y otras relacionadas con los riesgos para la salud de los operarios.

Desarrollar actividades de vigilancia epidemiológica de enfermedades profesionales, patología relacionada con el trabajo y ausentismo por tales causas.

Desarrollar actividades de prevención de enfermedades profesionales, accidentes de trabajo y educación en la salud de empresarios y trabajadores, conjuntamente con el subprograma de Higiene Industrial y Seguridad Industrial.

Dar asesoría en toxicología industrial sobre los agentes de riesgo y en la introducción de nuevos procesos y sustancias.

Mantener un servicio oportuno de primeros auxilios.

Prestar asesoría en aspectos médicos laborales, tanto en forma individual como colectiva.

Determinar espacios adecuados para el descanso y la recreación como medios para la recuperación física y mental de los trabajadores.

\section{La problemática del estrés laboral}

El mundo actual implica una época de grandes cambios, con ritmos de vida enormemente acelerado, mayor demanda de competencia y especialización. Continua reformulación de objetivos, metas, estrategias; entre otros aspectos. Este entorno exige a las personas mayor grado de autonomía, flexibilidad, capacidad de iniciativa, seguridad en sí mismo y capacidad para moldearse a situaciones nuevas. Precisamente las contrariedades y exigencias que cotidianamente debe enfrentar el hombre propician estar sometido al estrés y sus posibles consecuencias negativas.

$\mathrm{Al}$ estrés se le hace responsable de aspectos tan diversos como: la primera úlcera gástrica de un ejecutivo, el accidente de cierta persona, la incapacidad de un individuo para disfrutar de las relaciones sexuales con su pareja o su inexplicable depresión. De ahí que en la actualidad investigaciones multidisciplinarias se proyecten a su estudio desde la perspectiva de cómo controlarlo. 


\section{Acerca del estrés}

El uso del término estrés se ha popularizado sin que la mayoría de las personas tengan claro en qué consiste el mismo. Al revisar la amplia literatura sobre el tema, se encuentran multitud de definiciones, algunas de las cuales lo abordan indistintamente desde la perspectiva del estrés como estímulo, como respuesta o como consecuencia. Sin entrar a polemizar teóricamente sobre su definición, por no constituir objeto del presente trabajo, se aborda el estrés como: la respuesta adaptativa del organismo ante los diversos estresores, (Hans Selye, 1936).

Alternativamente para precisar conceptos, se utiliza el término "respuesta de estrés" al referirse a la respuesta inespecífica del organismo a cualquier demanda, y el término de "estresor" o "situación estresante" referida al estímulo o situación que provoca una respuesta de estrés.

En la actualidad han cobrado auge las teorías interaccionistas del estrés que plantean que la respuesta de estrés es el resultado de la interacción entre las características de la persona y las demandas del medio. Se considera que una persona está en una situación estresante o bajo un estresor cuando ha de hacer frente a situaciones que conllevan demandas conductuales que le resulta difícil poner en práctica o satisfacer. Es decir depende tanto de las demandas del medio como de sus propios recursos para enfrentarse a él (Lazarus y Folkman, 1984), o avanzando un poco más, de las discrepancias entre las demandas del medio externo o interno, y la manera en que el sujeto percibe que puede dar respuesta a esas demandas ( Folkman, 1984).

La respuesta de estrés es una respuesta automática del organismo a cualquier cambio ambiental, externo o interno, mediante la cual se prepara para hacer frente a las posibles demandas que se generan como consecuencia de la nueva situación, (Labrador, 1992). Por tanto, ello no es algo "malo" en sí mismo, al contrario; facilita el disponer de recursos para enfrentarse a situaciones que se suponen excepcionales.

Estas respuestas favorecen la percepción de la situación y sus demandas, procesamiento más rápido y potente de la información disponible, posibilitan mejor búsqueda de soluciones y la selección de conductas adecuadas para hacer frente a las demandas de la situación, preparan al organismo para actuar de forma más rápida y vigorosa. Dado que se activan gran cantidad de recursos (incluye aumento en el nivel de activación fisiológica, cognitiva y conductual); supone un desgaste importante para el organismo. Si éste es episódico no habrá problemas, pues el organismo tiene capacidad para recuperarse, si se repiten con excesiva frecuencia, intensidad o duración, pueden producir la aparición de trastornos psicofisiológicos. 


\section{Características de las situaciones estresantes}

Lazarus y Folkman (1984) señalan que existen ciertas características que parecen determinar el que una situación devenga como estresante, entre ellas se destacan: el cambio o novedad, la falta de información, la no predictibilidad, incertidumbre, ambigüedad, inminencia, duración y significación para la persona, entre otras. Al igual destacan las condiciones biológicas del organismo y la carencia de habilidades para hacerle frente.

Estos estresores pueden aparecer en diferentes contextos, ya sea laboral, familiar o social y pueden ser de tipo biogénico o psicosociales. Ambos tipos de estresores provienen tanto de estímulos externos a la propia persona como de aspectos internos.

Los estresores internos pueden ser estímulos de características físicas, como la sensación de malestar por mala digestión, el dolor producido por una herida, una enfermedad; o de características más cognitivas como el recuerdo de una situación desagradable, pensamientos sobre la propia inutilidad o lo difícil que puede ser el futuro inmediato, sentimientos de culpa, ambiciones no conseguidas.

Los estresores externos también pueden provenir de aspectos físicos como la temperatura ambiental extrema, ruido o la luz intensa; o implicar aspectos cognitivos, por ejemplo: ver que dos personas hablan en voz baja y de vez en cuando lo miran y se ríen. Aunque funcionan como estresores tanto los aspectos físicos como los cognitivos, estos últimos son más frecuentes e importantes.

\section{Fuentes de estrés}

Entre las variables contextuales generadoras de estrés se destacan el contexto laboral, el familiar y el social. Se tratará particularmente el estrés laboral. Consideraciones generales sobre el estrés laboral

A pesar de que muchas personas opinan que el trabajo no es una actividad gratificante en sí misma, lo cierto es que su ausencia total o el sólo temor de quedar desempleado con todas las posibles consecuencias que ello implica suelen propiciar la aparición de problemas psicológicos en el adulto. Numerosos estudios ponen de manifiesto cómo los individuos sin empleo muestran progresiva disminución de su implicación personal, progresivo proceso de desencanto y "desenganche social", fuertes sentimientos de humillación, resentimiento por la falta de ayudas, sentimiento de abandono por parte de los amigos y retraimiento social, al sentirse ciudadanos improductivos y dependientes (González Cabanach, 1998).

Sin embargo, también el trabajo puede provocar efectos negativos en las personas cuando no se organiza y estructura atendiendo a las necesidades y capacidades reales de los encargados de llevarlo a cabo, considerando como tal, al hombre en su sentido genérico. Es entonces cuando puede aparecer el estrés laboral.

El estrés laboral es uno de los problemas de salud más grave en la actualidad, que no sólo afecta a los trabajadores al provocarles incapacidad física o mental, sino también a los empleadores y los gobiernos, que comienzan a evaluar el perjuicio financiero que les causa el estrés. En los EUA, por ejemplo, su costo para la industria se calcula aproximadamente en 200000 millones de dólares anuales, debido al ausentismo, la merma en la productividad, las reclamaciones de indemnizaciones, los seguros de 
enfermedad y los gastos directos en servicios médicos. En el Reino Unido se calcula que el costo del estrés asciende cada año hasta el 10\% del PNB, como consecuencia de las enfermedades, la rotación del personal y la muerte prematura.

\section{Aspectos legales}

En EUA y algunos países europeos se ha constatado un incremento de las demandas legales de los trabajadores a las empresas en relación con daños producidos por el estrés laboral. De hecho, análisis realizado por el Consejo Nacional de Compañías de seguro, se estudiaron las demandas realizadas en 13 estados de los EUA y se encontró que las demandas por problemas mentales sin relación con daños físicos comenzaron a aparecer a finales de los años 70 y representaban el $2.5 \%$ de las demandas por enfermedad en 1983. Es significativo señalar que las mujeres representaban el 50\% de esas demandas entre 1980 y 1984, comparado con el 24\% de otro tipo de demandas. Las demandas provienen de múltiples niveles ocupacionales (directivos, supervisores, agentes de ventas, empleados, trabajadores); (National Council on Compensation Insurance, 1985).

En España se han producido cambios en la legislación sobre salud laboral con el fin de adecuarla a la normativa europea sobre esta materia. En ella se reconocen los posibles impactos negativos sobre la salud mental. El estudio detenido de esta nueva legislación y de la jurisprudencia que genere permitirá clarificar en qué medida el estrés es causa de enfermedad laboral y produce responsabilidades de las empresas por las que éstas tengan que compensar a sus trabajadores afectados.

\section{Reflexiones sobre el estrés laboral en cuba}

En la actualidad no cabe duda que el estrés laboral trae importantes consecuencias en el ámbito individual y organizacional. Bennis ha señalado que una organización saludable es aquella capaz de mantener el contacto adecuado con su entorno y de adaptarse al mismo, de desarrollar y mantener su sentido de identidad como organización imbuyéndolo en sus miembros, de potenciar y mantener la integración interna y de establecer la misión, estrategias y expectativas de desarrollo definidas. El deterioro de la calidad de vida laboral en determinada organización y los niveles elevados de estrés pueden repercutir negativamente sobre las contribuciones que sus recursos humanos han de realizar para preservar ese estado saludable. (Peiró, 1993).

En el actual nivel de desarrollo de la sociedad la actividad laboral se desempeña fundamentalmente en organizaciones constituidas por personas que se asocian para alcanzar objetivos determinados, esto provoca que la vinculación laboral con el entorno en que se enmarcan resulte estrecho y multifacético. Por otra parte uno de los objetivos básicos de la organización lo constituye el limar las tensiones que se producen entre sus miembros, las mismas pueden aparecer tanto por razones vinculadas a las tareas que se desempeñan como a aspectos de la multifacética y compleja vida social de los mismos.

Por otra parte el propio desarrollo social y tecnológico hacen cada vez más complejas las demandas tanto sobre la organización, en sentido de cumplir con sus objetivos, como de su relación con el medio, y para con sus integrantes. 
Cuba en las actuales condiciones resulta influida por los efectos de la crisis económica (período especial) que influye sobre el entorno laboral, como parte de la realidad social, haciéndola aún más compleja, por cuanto genera situaciones complejas para las organizaciones y sus recursos humanos, tanto en lo referido al cumplimiento de las tareas como por las tensiones internas que se generan y las características de las relaciones de la organización con su entorno. Al igual la necesidad de enfrentar la crisis económica provoca la adopción de distintos tipos de medidas tales como las de carácter organizativo que provocan por ejemplo redimensionamiento de organizaciones, cambios en la visión, misión, objetivos, y estrategias; aparición de nuevas organizaciones y nuevo tipos de estas (sector emergente); nuevas formas de propiedad como por ejemplo las empresas mixtas en algunos sectores y cooperativas de nuevo tipo en el sector agrícola; la inclusión de nuevas tecnologías que implican variaciones en las condiciones y exigencias laborales.

En resumen el trabajador se encuentra ante situaciones que resultan de alta demanda, en muchas ocasiones desconocidas, en cierto sentido inciertas, en algunos casos pueden provocar incertidumbre y desconfianza, o no sentirse totalmente preparado para enfrentarlas. Al igual el entorno social resulta complejo con situaciones nuevas cambiantes y de alta significación en aspectos de importancia cardinal para el desempeño de los distintos roles dentro de la sociedad.

La problemática descrita facilita la aparición de agentes estresantes o de convertirse en si mismas en propiciadoras de estrés. De ello deriva la particular importancia para la gestión de recursos humanos en las organizaciones cubanas actuales de brindar especial atención a aquellos factores capaces de convertirse en agentes estresores.

\section{El estrés laboral. Sus causas}

El estrés laboral aparece cuando como consecuencia del contenido o la intensidad de las demandas laborales o por problemas de índole organizacional, el trabajador comienza a experimentar vivencias negativas asociadas al contexto laboral, entre las que se destacan: apatía por el trabajo, astenia, dificultades en las relaciones interpersonales, disminución en el rendimiento laboral, tristeza, depresión, síntomas psicosomáticos que pueden llegar a generar la aparición de determinados trastornos psicofisiológicos, al igual que marcada insatisfacción laboral. (Se anexa encuesta para evaluar satisfacción laboral) (Rowshan, 1997).

Los factores estresantes pueden aparecer prácticamente en cualquier ocupación, profesión o puesto de trabajo y en cualquier circunstancia en que se somete a ese individuo a una carga a la que no puede ajustarse rápidamente. En definitiva, el estrés en el trabajo es un fenómeno tan variado y complejo como la propia vida.

González Cabanach (1998) distingue algunas de las fuentes potencialmente estresantes en el contexto laboral, entre ellas destaca:

Factores intrínsecos al propio trabajo, tales como: las condiciones físicas en que se realiza, la sobrecarga laboral, disponibilidad de recursos, el contenido de trabajo y otros. Factores relacionados con el desempeño de roles, por ejemplo: ambigüedad del rol, conflicto de roles, exceso o falta de responsabilidad, etc. 
Factores relacionados con las relaciones interpersonales que se generan en la vida laboral.

Factores relacionados con el desarrollo de la carrera profesional, por ejemplo: falta de congruencia, falta de competencia para desempeñar el puesto ocupado.

Factores relacionados con la estructura y el clima organizacional, como son: falta de participación en la toma de decisiones, carencia de autonomía, etc.

\section{Afrontamiento al estrés laboral}

Burke (1971), mediante entrevistas abiertas con personal directivo, identificó las conductas de afrontamiento ante situaciones de estrés categorizándolas en cinco grandes grupos: 1) hablar con otros; 2) trabajar más duro y durante más tiempo; 3) cambiar a una actividad de tiempo libre; 4) adoptar una aproximación de solución de problemas; y 5) alejarse de la situación estresante. Dewe et al., (1979), partiendo de conductas de afrontamiento realizadas por administrativos y trabajadores de oficina, obtuvieron cuatro tipos de estrategias: 1) acciones dirigidas a la fuente de estrés; 2) expresión de sentimientos y búsqueda de apoyo; 3) realización de actividades no laborales; y 4) intentos pasivos de esperar que pase la situación. Por su parte, Parasuraman y Cleek (1984) han distinguido entre respuestas adaptativas y no adaptativas de afrontamiento. Entre las primera incluían: planificar, organizar, priorizar tareas y conseguir apoyo de otros. Entre las segundas clasificaron las siguientes: trabajar más duro pero realizando más errores, hacer promesa poco realistas y evitar la supervisión.

Dewe y Guest (1990) han identificado siete tipos de estrategias de afrontamiento ante situaciones de estrés laboral, la primera de las cuales se centra en el problema y el resto en los aspectos emocionales: 1)abordar o trabajar sobre el problema; 2) intentar que el problema no se apodere de ti; 3) descarga emocional; 4) tomar medidas preventivas; 5) recuperarse y preparase para abordar en mejores condiciones el problema; 6) utilizar los recursos familiares y; 7) intentos pasivos de tolerar los efectos del estrés.

La tipología de afrontamiento desarrollada en el Organizational Stress Indicator (Cooper et al. 1988) distingue seis tipos de afrontamiento: 1) apoyo social; 2) estrategias referidas a la tarea; 3) lógica; 4) relaciones familiares y trabajo; 5) tiempo; y 6) implicación.

Estas tipologías plantean el problema del afrontamiento desde la perspectiva individual, sin embargo, en el ámbito del estrés laboral, numerosos autores han puesto de relieve la necesidad de contemplar diversos niveles de análisis: individual, grupal y organizacional.

A diferencia de lo que ocurre en otros ámbitos de la vida, las estrategias de afrontamiento consideradas en el ámbito laboral no resultan eficaces para reducir las relaciones entre estresores y sus consecuencias. Por cuanto la eficacia de las estrategias de afrontamiento depende de la posibilidad de control del estresor, y por ello las estrategias estudiadas, en situaciones de trabajo resultan ineficaces ya que el control de los estresores raras veces está en manos del individuo. La mayor parte de los estresores laborales están entre los que se caracterizan como poco adecuados para las soluciones individuales. Su afrontamiento más bien requiere esfuerzos cooperativos organizados 
que trascienden el nivel individual, no dependiendo su solución de la habilidad de la persona para manejar sus recursos individuales. Así pues, es necesario identificar las estrategias de afrontamiento grupales y organizacionales, por cuanto con frecuencia las situaciones de estrés provienen de esos niveles

Newman y Beehr (1979), señalan que las respuestas de afrontamiento en el trabajo requieren la implicación activa de la organización. Esta respuesta puede concretarse en rediseño de puestos, cambios en la estructura organizacional, "feedback" para contribuir a la clarificación del rol, redefinición de criterios de selección y ubicación, introducción de formación de relaciones humanas, mejora de las condiciones de trabajo, mejora de la comunicación y mejora de los beneficios para el trabajador así como la introducción de servicios de promoción de salud.

\section{Efectos negativos del estrés en el trabajador}

La exposición a situaciones de estrés no es en sí misma algo "malo" o que conlleve a efectos necesariamente negativos, solo cuando las respuestas de estrés son excesivamente intensas, frecuentes y duraderas pueden producirse diversos trastornos en el organismo. En los momentos iniciales estos trastornos son relativamente leves, lo cual se debe a que antes que se desarrolle un trastorno importante, el organismo emite señales que permiten ponerse en guardia y prevenir el desarrollo de problemas más importantes. ( Valdés, 1995).

Entre los principales efectos se destacan:

Fisiológicos: Aumento de la tasa cardiaca, la presión arterial, la sudoración, del ritmo respiratorio, la tensión muscular, así como de los niveles de adrenalina y noradrenalina. Incremento de los niveles de azúcar en la sangre. Disminución del riego sanguíneo periférico y de la actuación del sistema digestivo. Incremento del metabolismo basal, del colesterol y liberación de ácidos grasos en la sangre. Aumento de los niveles de corticoides. Inhibición del sistema inmunológico. Dificultad para respirar. Sensación de nudo en la garganta. Sequedad en la boca. Dilatación de las pupilas.

Cognitivos: Preocupaciones. Dificultad para la toma de decisiones. Sensación de confusión. Incapacidad para concentrarse. Dificultades para dirigir la atención. Sentimiento por falta de control. "Estrechamiento" de la atención. Desorientación. Olvidos frecuentes. Bloqueos mentales. Hipersensibilidad a la crítica.

Motores: Hablar rápido. Temblores. Tartamudeo. Voz entrecortada. Imprecisión. Precipitaciones. Explosiones emocionales. Predisposición a accidentes. Consumo de drogas (psicofármacos, alcohol, café). Comer en exceso o inapetencia. Bostezos. Trastornos del sueño. 


\begin{tabular}{|c|c|c|}
\hline \multicolumn{3}{|c|}{ Factores causantes del estrés laboral. } \\
\hline Causas & Factores influyentes & Consecuencias posibles \\
\hline $\begin{array}{l}\text { Condiciones de } \\
\text { Trabajo }\end{array}$ & $\begin{array}{l}\text { Sobrecarga cuantitativa de trabajo. } \\
\text { Sobrecarga cualitativa de trabajo. } \\
\text { Decisiones comunes. } \\
\text { Peligro Físico. } \\
\text { Trabajo por turnos. }\end{array}$ & $\begin{array}{l}\text { Fatiga física o mental. } \\
\text { Agotamiento del sistema nervioso. } \\
\text { Irritabilidad. } \\
\text { Tensión nerviosa. }\end{array}$ \\
\hline Papel desempeñado & $\begin{array}{l}\text { Ambigüedad de los papeles. } \\
\text { Sexismo. } \\
\text { Hostigamiento sexual. }\end{array}$ & $\begin{array}{l}\text { Ansiedad y tensión nerviosa. } \\
\text { Disminución del rendimiento. }\end{array}$ \\
\hline $\begin{array}{l}\text { Factores } \\
\text { interpersonales }\end{array}$ & $\begin{array}{l}\text { Mal sistema de trabajo y falta de } \\
\text { apoyo social. } \\
\text { Rivalidades Políticas. } \\
\text { Celos profesionales. } \\
\text { Falta de atención a los trabajadores } \\
\text { por parte de la organización. }\end{array}$ & $\begin{array}{l}\text { Insatisfacción profesional. } \\
\text { Tensión nerviosa. } \\
\text { Hipertensión. }\end{array}$ \\
\hline $\begin{array}{l}\text { Progresión } \\
\text { profesional }\end{array}$ & $\begin{array}{l}\text { Avance demasiado lento. } \\
\text { Avance demasiado rápido. } \\
\text { Seguridad del empleo. } \\
\text { Ambiciones frustradas }\end{array}$ & $\begin{array}{l}\text { Baja de la productividad. } \\
\text { Pérdida de la confianza en si mismo. } \\
\text { Irritabilidad e ira. }\end{array}$ \\
\hline Estructura orgánica & $\begin{array}{l}\text { Estructura rígida e impersonal. } \\
\text { Discusiones políticas. } \\
\text { Una supervisión o formación } \\
\text { inadecuadas. } \\
\text { Imposibilidad de participar en la } \\
\text { toma de decisiones. }\end{array}$ & $\begin{array}{l}\text { Insatisfacción profesional. } \\
\text { Pérdida de motivación. } \\
\text { Baja productividad. }\end{array}$ \\
\hline $\begin{array}{l}\text { Relación hogar- } \\
\text { trabajo. }\end{array}$ & $\begin{array}{l}\text { Repercusiones de la vida laboral en } \\
\text { la familia. } \\
\text { Falta de apoyo del cónyuge. } \\
\text { Peleas domésticas. } \\
\begin{array}{l}\text { Estrés provocado por una doble } \\
\text { carrera. }\end{array}\end{array}$ & $\begin{array}{l}\text { Conflictos psicológicos y fatiga mental. } \\
\text { Falta de motivación y disminución de la } \\
\text { productividad. } \\
\text { Recrudecimiento de las peleas } \\
\text { domésticas. }\end{array}$ \\
\hline
\end{tabular}




\section{Consecuencias del estrés para la organización:}

Cada persona que sufre de estrés está pagando un alto precio por su salud personal, pero también pagan un alto costo la empresa para la cual trabaja y la economía nacional pues trae como consecuencia:

Absentismo.

Rotación o fluctuación del personal.

Disminución del rendimiento físico.

Disminución del rendimiento psicológico.

Afectaciones en la calidad del trabajo realizado.

Accidentes.

Indemnizaciones por conceptos de reclamación o certificados médicos.

Otras.

Cooper (1983) señala en la siguiente tabla algunas de las principales causas del estrés laboral y las posibles consecuencias que este puede generar.

Técnicas para el control del estrés laboral

La lucha contra el estrés laboral constituye uno de los grandes empeños que deberán acometer tanto los gobiernos, como las estructuras de dirección en los diferentes niveles y los sindicatos en los próximos años. Las empresas que probablemente tengan más éxitos en el futuro serán las que ayuden a los trabajadores a hacer frente al estrés y adapten las condiciones y la organización del trabajo a las actitudes humanas.

Los elementos abordados teóricos y metodológicos abordados y las referencias empíricas con que se cuentan en la actualidad dejan por sentado que el fenómeno del estrés laboral es perfectamente controlable.

Al trabajar el estrés laboral debe tomarse en cuenta que su control debe trascender necesariamente el límite de lo individual y considerar lo grupal y lo organizacional. Ha habido muchas formas de clasificar las intervenciones en este sentido Mattenson e Ivanevich (1987) distinguen entre técnicas y estrategias preventivas y curativas; DefranK y Cooper (1987) sugieren que las intervenciones pueden atender a aspectos individuales, organizacionales o de la "interfase" entre individuo y organización y Murphy (1988) diferencia tres niveles de intervención: primaria (reducción de los estresores), secundaria (gestión o manejo del estrés) y terciaria (programas de asistencia a los empleados).

A continuación se presentan algunas sugerencias de estrategias válidas para el control del estrés laboral desde dos perspectivas:

Estrategias de intervención a nivel organizacional.

Estrategias de intervención a nivel individual. 


\section{Estrategias de intervención en el ámbito organizacional.}

Al enfocar el enfrentamiento al estrés es importante tener en cuenta que el mismo debe ser enfrentado desde dos perspectivas: la individual y la organizacional.

Para abordar la forma en que las organizaciones deben enfrentar el estrés laboral es necesario considerar que cada organización, por poseer características que la hacen particular, debe adoptar formas de enfrentamiento acorde a la cultura que prevalece en la misma; a la par que por cuanto la organización se encuentra imbricada en un sistema de relaciones con otras organizaciones y con la sociedad en sentido general existe un conjunto de variables que no pueden ser controladas sólo por la misma, que pueden contribuyen a la aparición de este.

Por tanto desarrollar estrategias para la disminución del estrés laboral implica, tener en cuenta la cultura de la organización, (nivel arquitectónico, valores y presunciones básicas que operan en la misma). Al igual evaluar variables que puedan influir en el comportamiento de las personas y grupos que se desarrollan en una organización tales como: estilos de dirección, liderazgo, comunicación organizacional e interpersonal, clima sociopsicológico, estilos de solución de conflictos, distribución de funciones y claridad de las mismas, organización y diseño de los puestos de trabajo, satisfacción laboral, motivación, entre otras.

El diagnóstico de estas variables no es estático, debe buscarse la explicación del funcionamiento de ellas en la organización y como los individuos operan bajo los efectos de las mismas.

Una organización en que su sistema de gestión de recursos humanos considere al hombre el centro de todos los procesos que se dan hacia dentro y fuera de la misma traza y planifica estrategias en diferentes direcciones teniendo en cuenta las variables anteriormente mencionadas.

Para la concepción y aplicación de estas estrategias resultan necesarios entre otros los siguientes aspectos:

Estudios de puestos de trabajo, que establezcan las exigencias del mismo y por consiguiente encuentren los efectos negativos del trabajo sobre el hombre que desempeñará esas funciones, entre ellos el estrés laboral.

Diseño y puesta en marcha de sistemas de selección de recursos humanos que tomen en cuenta los efectos negativos de la relación del hombre con su actividad laboral, que permitan seleccionar personas menos vulnerables al estrés que pueda generar el puesto, que se caractericen por la flexibilidad en sus estilos comunicativos y de manejo de los conflictos, o en su defecto, detectar la vulnerabilidad de estos sujetos y trabajar profesionalmente sobre los mismos. Implementar sistemas de capacitación en los que además de las necesidades de aprendizajes detectadas se desarrollen el conjunto de competencias laborales y sociales que permitan potenciar y fortalecer a los miembros de la organización. Promover y tomar en cuenta las habilidades necesarias para asumir los distintos cargos y dar seguimiento a quienes los ocupan, previendo los posibles estresores. 
Realizar evaluaciones frecuentes a las personas que ocupan puestos capaces de generar efectos negativos. Tener en cuenta factores o grupos de riesgos tales como: embarazadas, personal de edad avanzada, que hayan padecido enfermedades y otros.

Implementar entrenamientos, y/o sesiones de relajación antes, durante y después de la jornada laboral, proponer y facilitar la realización acciones para el uso del tiempo libre y desarrollo de adecuadas relaciones interpersonales.

Estas propuestas pueden considerarse estrategias de carácter general a seguir por la organización, lo idóneo para la realización de las mismas es que la organización sea vista como un sistema y que no se planifique por tanto de forma aislada las acciones, pues no alcanzarían los resultados esperados y necesarios para la organización y los sujetos que la conforman.

Tratando de responder la pregunta inicial y para ser consecuentes con la teoría psicológica marxista del desarrollo de la personalidad, la organización por sí sola, aún implementando las mejores acciones para la disminución del estrés, no tendrá resultado feliz si el individuo no se compromete y establece nuevos estilos de vida que le permitan asumir el reto de aprender a manejar el estrés, por lo que es importante proponer vías para el enfrentamiento individual.

\section{Estrategias de intervención a nivel personal.}

Cómo enfocar la vida

El trabajo debe, antes que todo, darnos satisfacción y tener objetivos viables para cada persona, siempre dentro del área más afín con las preferencias personales, bien sea en los negocios, el arte, la ciencia, la oficina, el taller, etc.

Además, el nuevo enfoque de la vida que estamos sugiriendo debe hacer balance entre lo que cada quien procura para sí y lo que procura para los demás, para lo que se deben observar algunas recomendaciones útiles a estos efectos:

No ser perfeccionista, pues la perfección es imposible. Si alguien tiene impulsos perfeccionistas, debe adoptar una actitud de continuo desengaño ante esta utopía fatalista.

Finalmente recordar que todos somos diferentes y que aquello que funciona para otros no necesariamente funciona para uno. Por ello cada quien necesita su propio estilo de comportamiento ante la vida

Hay que optar por el camino de la simplicidad, pues resulta más fácil enfrentarse con las vicisitudes de la vida si evitamos las complicaciones innecesarias.

Preguntémonos si no nuestros problemas son en realidad críticos, en no pocas ocasiones tras el análisis encontramos que no es para tanto.

Pensar positivamente, vivir en forma negativa, bajo un criterio trágico produce muchas zozobras. Es oportuno contemplar el lado positivo de la vida, aunque se sienta que se falla en el intento.

No postergar enfrentarse a problemas molestos, vale más sumergirse en ellos para obtener lo mejor que sea posible, que estar a la expectativa, pues esto genera más ansiedad. 
No permitamos que la falta de éxito nos desvaste, tengamos en cuenta que nadie actúa de forma perfecta, siempre existe más perdedores que triunfadores, más dirigidos que dirigentes. Si damos lo mejor ante cada situación no debe abatirnos no alcanzar totalmente los objetivos.

\section{Estrategias para encontrarse uno mismo}

Evite estar demasiado tiempo rumiando sus problemas personales, magnificándolos y cayendo en un círculo de autocompasión, autodegradación e improductividad personal.

Sea consciente de las causas de sus sentimientos de soledad, aprenda a identificar su raíz causal antes de que estos ocurran. La sola conciencia de este proceso le ayudará emocionalmente a manejarlo.

Desarrolle descargas constructivas, creativas. Haga cualquier cosa que estimule que estimule su pensamiento y le permita concentrarse en algo fuera de usted mismo. Si no tiene una profesión o trabajo realice labor filantrópica.

Trate de ver las cosas positivas de su vida pues las ha de tener, pero si cree que no posee ninguna, piense que el sólo hecho de estar vivo y sano es una bendición

Cultive su capacidad para encontrar gozo de la vida cotidiana. Tómese tiempo para apreciar y gozar la gran variedad de paisajes, sonidos y situaciones que le rodean. Paseé por un parque, escuche un concierto, desarrolle actividades con sus amigos, etc.

Sea agresivo en su actitud ante la vida involúcrese en algo emocionante y constructivo.

Evite caer en sentimiento de vacío y temor ante el futuro. Identifique y dele las perspectivas oportunas a cada temor, pues la incertidumbre es el enemigo oculto más peligroso.

Aprenda a recompensarse, por lo general somos generoso para los demás, pero para nosotros mismos.

Haga algo por ayudar a otra persona. Ponerse al servicio de otro pues genera le ayudará a salir de sus propios problemas, los sacará de su mente.

Comprométase en actividades físicas, el ejercicio es sumamente terapéutico pues genera sentimientos placenteros que inciden en su estado de ánimo.

Busque personas que deseen escucharle o ayudarle, evite aquellas que resaltan sus frustraciones y promueven su hundimiento.

No realice nada que le haga sentir peor.

Sea su propio amigo y así tendrá un amigo toda la vida. Si Ud. se aprecia lo demás lo apreciarán, si se ama los demás lo amarán

No piense que sentirse sólo y triste es una debilidad. Piense que ello es parte del ser humano.

Conozca el placer de su propia compañía y de esta forma descubrirá el centro de su propio ser 


\section{La meditación}

Se entiende por meditación un estado de conciencia provocado por diversas técnicas que buscan separar un poco al individuo de su vida diaria, disminuyendo su conciencia analítica normal y logrando una "perspectiva de unidad"

La persona que medita sigue en general procedimientos especiales o realiza determinados ejercicios para lograr dicho estado. Los ejercicios de meditación pueden ser de varios tipos. En la meditación denominada concentrativa se intenta restringir la conciencia a una sola fuente invariable de estimulación durante cierto tiempo. Los que meditan pueden concentrarse mirando un objeto, poniendo atención en algún proceso como la respiración, escucharse a sí mismo cantando en voz alta, o simplemente repitiendo silenciosamente una palabra o una frase.

En el estado de meditación concentrativa cesan todas las percepciones y pensamientos activos de naturaleza analítica.

Esta sensación no dura más que algunos minutos, pero aparece como un fenómeno desligado del tiempo para el participante, quién después se siente renovado y mejor capacitado para experimentar la vida directamente.

¿Qué decir de los efectos fisiológicos de la meditación? ¿De cómo la meditación reduce el strees?

Los investigadores Wallace y Benson, sometieron a estudios de laboratorios a un grupo de meditadores y encontraron que durante el acto de la meditación el cuerpo humano se caracteriza por una pauta de actividad ambivalente: alerta, pero al vez muy relajada. Específicamente el ritmo metabólico del cuerpo se torna insólitamente bajo y, en consecuencia, el consumo de oxigeno, la eliminación de bióxido de carbono y el volumen y ritmo de respiración reducido. El ritmo cardíaco también disminuye significativamente y la producción de sustancias químicas en la sangre (lactato de sodio) muestra un marcado descenso, su exceso en sangre se asocia a sentimientos de angustia.

Por su parte en la actividad eléctrica del cerebro predomina el ritmo alfa indicador de máximo reposo, que además se relaciona con sentimientos de placidez como si el cuerpo estuviera flotando en un ambiente de mucha paz, con sensación de descanso y comodidad.

\section{Cómo meditar}

Es esencial contar con ambiente tranquilo, exento de ruido proveniente del exterior, se puede usar música suave como fondo.

En el ambiente físico así logrado se procede a tranquilizar el ambiente interno, la mente debe estar libre provenientes de otras partes del cuerpo, incluyendo la musculatura. La sesión debe durar de 20 a 30 minutos.

La relajación muscular

Es capaz de propiciar una serie de cambios físicos que contrarrestan el stress. Estos cambios incluyen, como en el caso de la meditación, disminución del ritmo cardíaco y de la tensión muscular, así como reducción de la presión arterial y del ritmo de la respiración. Pero sobre todo provoca disminución de la tensión mental y sensación placentera, tanto mental como física. 
Relajarse es una habilidad y como otras habilidades requiere aprenderla, así como disciplina regularidad para practicarla. Es recomendable realizar dos sesiones diarias de 30 minutos cada una.

\section{Referencias bibliográficas}

Chiavenatto, I(1992) Administración de Recursos Humanos. México: Mc Graw-Hill González Cabanach, R (1998) " Comunicación, estrés y accidentabilidad. Tres factores de actualidad". ¿?

Labrador, F. J. (1992) El estrés: Nuevas Técnicas para su control. Temas de Hoy. Madrid. España.

Lazarus y Folkman, (1986) Estrés y procesos cognitivos. Editorial Martínez Roca. Barcelona, España.

Peiró, JUL (1993) Desencadenantes del estrés laboral. Serie Recursos Humanos.. EUDEMA, S.A. (Ediciones de la Universidad Complutense, S.A) Madrid, España.

Rowshan, A (1997) El estrés. Técnicas positivas para atenuarlo y asumir el control de tu

Selye, H (1976). The stress of life. McGraw-Hill (edición revisada). New York

Vida. Ediciones. ONIRO, S.A. Barcelona, España.

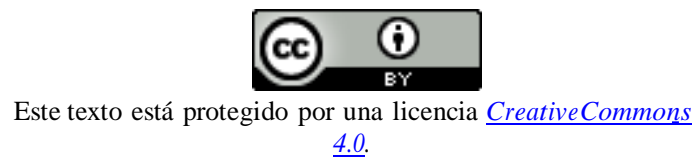

Usted es libre para Compartir - copiar y redistribuir el material en cualquier medio o formato - y Adaptar el documen- to -remezclar, transformar y crear a partir del material- para cualquier propósito, incluso comercialmente, siempre que cumpla la condición de:

Atribución: Usted debe reconocer el crédito de una obra de manera adecuada, proporcionar un enlace a la licencia, e in- dicar si se han realizado cambios. Puede hacerlo en cualquier forma razonable, pero no de forma tal que sugiera que tie- ne el apoyo del licenciante o lo recibe por el uso que hace. 